Rubincam 11 April 2000

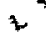

\title{
Pluto and Charon:
}

\section{A case of precession-orbit resonance?}

\author{
by
}

\author{
David Parry Rubincam \\ Geodynamics Branch, Code 921 \\ Laboratory for Terrestrial Physics \\ NASA's Goddard Space Flight Center \\ Greenbelt, MD 20771 \\ voice: $301-614-6464$ \\ fax : 301-614-6522 \\ email: rubincam@denali.gsfc.nasa.gov
}

11 April 2000 


\section{Abstract}

Pluto may be the only known case of precession-orbit resonance in the solar system. The Pluto-Charon system orbits the Sun with a period of 1 Plutonian year, which is 250.8 Earth years. The observed parameters of the system are such that Charon may cause Pluto to precess with a period near 250.8 Earth years. This gives rise to two possible resonances, heretofore unrecognized. The first is due to Pluto's orbit being highly eccentric, giving solar torques on Charon with a period of 1 Plutonian year. Charon in turn drives Pluto near its precession period. Volatiles, which are expected to shuttle across Pluto's surface between equator and pole as Pluto's obliquity oscillates, might change the planet's dynamical flattening enough so that Pluto crosses the nearby resonance, forcing the planet's equatorial plane to depart from Charon's orbital plane. The mutual tilt can reach as much as $2^{\circ}$ after integrating over $5.6 \times 10^{6}$ years, depending upon how close Pluto is to the resonance and the supply of volatiles. The second resonance is due to the Sun's traveling above and below Charon's orbital plane; it has a period half that of the eccentricity resonance. Reaching this half-Plutonian year resonance requires a much larger but still theoretically possible amount of volatiles. In this case the departure of Charon from an equatorial orbit is about $1^{\circ}$ after integrating for $5.6 \times 10^{6}$ years. The calculations ignore libration and tidal friction. It is not presently known how large the mutual tilt can grow over the age of the solar system, but if it remains only a few degrees, then observing such small angles from a Pluto flyby mission would be difficult. It is not clear why the parameters of the Pluto-Charon system are so close to the eccentricty resonance. 


\section{Introduction}

Pluto may be the only known case of precession-orbit resonance in the solar system, with the planet being near a resonance associated with the eccentricity of its orbit about the Sun. Volatiles moving about on Pluto's surface may cause the planet to sweep across this resonance.

The mechanism is the following. Pluto has an equatorial bulge caused by rotational flattening and the tidal attraction of Charon. Charon's gravitational torque on this bulge would attempt to make Pluto precess on Charon's orbital plane if Pluto's spin axis were inclined to it. Due to the nearness of Charon to Pluto, this precession period is very short. The precession period in fact appears to be close to the orbital period of the system about the Sun, which is 250.8 Earth years = one Plutonian year.

Charon revolves about Pluto with a period of 6.38726 days, which is short compared to the motion of the system about the Sun; so that in effect the Sun sees a ring about Pluto. The Sun torques this ring with periodicities of one Plutonian year due to the eccentricity ( $e=0.244$ ) of the orbit about the Sun. Pluto's flattening effectively makes a ring of matter around Pluto's equator, with the ring from Charon torqueing Pluto's ring with the same periodicies as the solar torque on the system. Because Charon is so close to Pluto, this torque is strong. This torque is capable of inclining Pluto's equator to Charon's orbital plane if the system is close to the eccentricity resonance.

Pluto does in fact appear to be very close to the eccentricity resonance. The volatiles nitrogen and methane, which may move from equator to pole as Pluto's obliquity varies, could change Pluto's flattening enough to drive the planet across the eccentricity resonance, forcing Pluto to tip slightly relative to Charon's orbital plane. In other words, Charon no longer orbits in Pluto's equatorial plane. The amount of tilt is small: on the 
order of $2^{\circ}$ at most after two obliquity cycles $\left(5.6 \times 10^{6}\right.$ years $)$, depending on Pluto's nearness to the resonance and the supply of volatiles.

Another possibility is that Pluto crosses the resonance associated with half the period of the orbit about the Sun (125.4 Earth years = half a Plutonian year) as the Sun's travels above and below Charon's orbital plane. The amount of volatiles moving between equator and pole over the obliquity cycle would have to be larger than in the case of the eccentricity resonance, but is still theoretically possible. The amount of mutual tilt after 5.6 $\times 10^{6}$ years on the order of $1^{\circ}$.

\section{Pluto's precession}

This section demonstrates that a hydrostatic Pluto is near the eccentricity resonance for the parameters of the Pluto-Charon system derived from astronomy. That Pluto can reach this resonance and perhaps the half-Plutonian year resonance is shown in Section 3.

Charon causes Pluto to precess about their mutual orbital plane with a period $T$ of approximately

$$
T \cong \frac{2 \pi H}{L}
$$

where $H$ is Pluto's rotational angular momentum

$$
H=C_{p} \omega_{P}=\lambda_{P} M_{P} R_{P}^{2} \omega_{P}
$$

and $L$ is 


$$
L=\frac{3 G\left(M_{P}+M_{C}\right) M_{C} J_{2}^{P} R_{P}^{2}}{2 a^{3}}=\frac{3 n^{2} M_{C} J_{2}^{P} R_{P}^{2}}{2}
$$

[e.g., Goldreich, 1966], with the measure of the moment of inertia being

$$
\lambda_{P}=\frac{C_{P}}{M_{P} R_{P}^{2}} .
$$

In these equations $C_{P}$ is Pluto's moment of inertia about its rotational axis, $\omega_{P}$ the planet's rotational speed, $M_{P}$ its mass, $R_{P}$ its radius, $\lambda_{P}$ its condensation factor, and $J_{2}^{P}$ its second degree zonal coefficient in the spherical harmonic expansion of Pluto's gravitational field; while $M_{C}$ is Charon's mass, $a$ is Charon's distance from Pluto, $n$ is Charon's mean motion, and $G$ is the universal constant of gravitation. Because of observed synchronous rotation $\omega_{P}=n$, where

$$
n^{2}=G\left(M_{P}+M_{C}\right) / a^{3}
$$

The $M_{P}+M_{C}$ term in $L$ may look peculiar, but it is correct and comes from having two masses accelerating, instead of one fixed mass with the other accelerating around it [Brouwer and Clemence, 1961, pp. 268-272].

$J_{2}^{P}$, which is a measure of the flattening of the planet, will be taken in this section to be the hydrostatic value. The flattening is due to both rotation about its axis and to tides from Charon [Bursa, 1994], so that

$$
J_{2, \text { hydro }}^{P}=J_{2, \text { rot }}^{P}+J_{2, \text { tides }}^{P} .
$$


For a rotating planet in hydrostatic equilibrium, the response due to the rotational potential

$$
V_{\text {rot }}=-\omega_{P}^{2} r^{2} P_{20}(\cos \alpha) / 3
$$

is approximately

$$
J_{2, \text { rot }}^{P}=\frac{\omega_{P}^{2} R_{P}^{3}}{3 G M_{P}}\left[\frac{5}{1+\left(\frac{5}{2}-\frac{15 \lambda_{P}}{4}\right)^{2}}-1\right]
$$

where $r$ is the distance from Pluto's center and $\alpha$ is the colatitude measured from the rotation axis [e.g., Kaula, 1968, pp. 68-73]. Values for $J_{2, \text { rot }}^{P}$ are on the order of $1 \times 10^{-4}$. For the equilibrium tides Pluto responds to Charon's tidal potential

$$
V_{\text {tides }}=\frac{G M_{C} r^{2}}{a^{3}}\left[\frac{3}{2} \cos ^{2} \Psi-\frac{1}{2}\right]
$$

where $\Psi$ is the angle measured from the line joining Pluto and Charon. This line lies nearly in Pluto's equator, so that the $P_{20}(\cos \alpha)$ component introduces a factor of one-half by the addition theorem [e.g., Kaula, 1968, p. 67]. Therefore 


$$
J_{2, \text { tides }}^{P}=\frac{3 G M_{C}}{2 \omega_{P}^{2} a^{3}} J_{2, \text { rot }}^{P}=\frac{1}{2}\left(\frac{R_{P}}{a}\right)^{3}\left(\frac{M_{C}}{M_{P}}\right)\left[\frac{5}{1+\left(\frac{5}{2}-\frac{15 \lambda_{P}}{4}\right)^{2}}-1\right]
$$

which is about $15 \%$ or so of the hydrostatic value and forms a non-trivial component of the flattening. Substituting (2) through (10) in (1) and remembering that $\omega_{P}=n$ give

$$
T=\left[\frac{\left(\frac{8 \pi}{n}\right)\left(\frac{a}{R_{P}}\right)^{3}}{2\left(\frac{M_{C}}{M_{P}}\right)\left(1+\frac{M_{C}}{M_{P}}\right)+3\left(\frac{M_{C}}{M_{P}}\right)^{2}}\right] \cdot\left[\frac{\lambda_{P}}{\frac{5}{1+\left(\frac{5}{2}-\frac{15 \lambda_{P}}{4}\right)^{2}}-1}\right]
$$

The only parameter which is well-determined in this equation is the mean motion $n$, which is obtained from light curves; the motion of Charon and Pluto about their center of mass has a period of $6.38726 \pm 0.00007$ days [Tholen and Tedesco, 1994; Tholen and Buie, 1997, p. 195]. Estimates for $a$ vary between $19,405 \mathrm{~km}$ and $19,662 \mathrm{~km}$ [Null et al., 1993; Null and Owen, 1996], while the estimates of $R_{p}$ range from $1100 \mathrm{~km}$ to $1206 \mathrm{~km}$ [McKinnon et al., 1997, p. 298]. The measurements based on mutual events and direct imaging, which appear to give the most accurate results, put $R_{P}$ between about $1150 \mathrm{~km}$ and $1200 \mathrm{~km}$; this is the range which will be adopted here. The ratio $a / R_{p}$ is thus assumed to lie between 16.2 to 17.1 .

Much more poorly determined are $M_{C} M_{P}$ and $\lambda_{P}$. Values for $M_{C} M_{P}$ run from 0.0837 to 0.1566 [Null et al., 1993; Young et al., 1994], varying by almost a factor of 2. This range will be assumed here. 
The condensation factor $\lambda_{P}$ depends upon the density distribution inside Pluto, which is unknown. From the mass and size estimates Pluto appears to be about $50 \%-70 \%$ rock and 30\%-50\% ice [McKinnon et al., 1997]. If Pluto has not differentiated, then $\lambda_{p}$ can be expected to be near 0.4 , the value for a planet with constant density throughout the interior. On the other hand, Pluto may have differentiated into a rocky core surrounded by an icy mantle. If the core has a constant density $\rho_{r}$ and the mantle a constant density $\rho_{i}$, then the condensation factor (4) can be shown to be

$$
\lambda_{P}=\frac{C_{P}}{M_{P} R_{P}^{2}}=\frac{2}{5}\left[\frac{\left(\bar{\rho}-\rho_{i}\right)^{5 / 3}}{\bar{\rho}\left(\rho_{r}-\rho_{i}\right)^{2 / 3}}+\frac{\rho_{i}}{\bar{\rho}}\right]
$$

where $\bar{\rho}$ is the average density of the whole planet. For ice with density $\rho_{i}=1000 \mathrm{~kg} \mathrm{~m}^{-3}$ and anhydrous rock with density $\rho_{r}=3500 \mathrm{~kg} \mathrm{~m}^{-3}, \lambda_{P}$ could be as low as 0.305 , depending upon the value of $\bar{\rho}$. On the other hand, for hydrated rock with density $\rho_{r}=$ $2800 \mathrm{~kg} \mathrm{~m}^{-3}$ [McKinnon et al., 1997, p. 302], $\lambda_{P}$ could only get as low as 0.322 . Thus a reasonable range for $\lambda_{P}$ is probably 0.305 to 0.400 .

Given the uncertainties in $a / R_{p}, M_{C} M_{p}$, and $\lambda_{p}$, the best strategy appears to be to assume Pluto is in one of the two resonances and plot $M_{C} M_{P}$ vs. $\lambda_{P}$ to see whether the parameters appear reasonable. This is easily done; (11) is quadratic in $M_{C} M_{p}$, giving 


$$
\frac{M_{C}}{M_{P}}=\frac{-2+\sqrt{4+\frac{160 \pi \Gamma}{n T}\left(\frac{a}{R_{P}}\right)^{3}}}{10}
$$

where $\Gamma$ is the right-hand factor in square brackets in (7)

$$
\Gamma=\frac{\lambda_{P}}{1+\left(\frac{5}{2}-\frac{15 \lambda_{P}}{4}\right)^{2}}-1
$$

and depends only on $\lambda_{p}$.

The results are plotted in Figure 1. The lower shaded area shows the range for the eccentricity resonance, while the upper shaded area does the same for the half-Plutonian year resonance. The lower boundary is at $\left(a / R_{P}\right)=16.2$ and the upper boundary is at 17.1 for each resonance. The resonances lie somewhere in the shaded areas. The region between the dashed lines shows the observed range of $M_{C} M_{P}$, which is 0.0837 to 0.1566 . The value for $n$ is assumed to be $2 \pi /(6.38726$ days $)$, while the Plutonian year is taken to be 250.8 Earth years [Malhotra and Williams, 1997]. The lower shaded area in Figure 1 shows that resonant values of $M_{C} M_{P}$ fall within the observed range for the larger values of $\lambda_{p}$. Hence a hydrostatic Pluto is near the eccentricity resonance, but not near the halfPlutonian year resonance. The half-Plutonian year resonance (upper shaded area) appears to be ruled out unless the observations are badly in error and Charon is much more massive than presently thought, or Pluto has a large nonhydrostatic component to its flattening. That a nonhydrostatic Pluto can reach both resonances through the transport of volatiles is demonstrated in Section 4. 
Given the wide range of possible values for ratios of moon to planetary masses, the nearness to the eccentricity resonance may not be coincidental. Hence the Pluto-Charon system might actually be in this resonance, but is probably not near the half-Plutonian year resonance. For arguments against being in the eccentricity resonance, see the Discussion.

\section{Dynamics}

Because of the similarity to the Earth-Moon-Sun system, Goldreich's [1966] equations can be adapted to the Pluto-Charon-Sun system. Charon and the Sun are considered to be point-masses. Charon is assumed to be in a circular orbit about Pluto. The equations average over the orbital motion of Charon about Pluto, which has a short period (6 days) compared to the motion of the system about the Sun (250 years).

Goldreich's equations, however, are to be modified in the following ways: (1) allow the Pluto-Charon system to be in an eccentric orbit about the Sun so as to be able to compute the solar torques which vary with solar distance; and (2) marry Goldreich's equations with Ward's [1974] so as to include the precession of the orbit on the invariable plane. The variables will be Ward's: obliquities and precession angles measured with respect to the Pluto-Charon system's orbit about the Sun.

Let $\hat{\mathbf{x}}, \hat{\mathbf{y}}$, and $\hat{\mathbf{z}}$ be unit vectors defining a right-handed inertial coordinate system where $\hat{\mathbf{z}}$ is normal to the solar system's invariable plane (see Figure 2). Let $\hat{\mathbf{n}}$ be the unit vector normal to the orbit of the Pluto-Charon system about the Sun, so that

$$
\hat{\mathbf{n}}=(\sin I \sin \Omega) \hat{\mathbf{x}}-(\sin I \cos \Omega) \hat{\mathbf{y}}+(\cos I) \hat{\mathbf{z}}
$$


where $I$ is the inclination of the orbit to the invariable plane and $\Omega$ is the angle of the ascending node in the invariable plane. The unit vector $\hat{\mathbf{x}}^{\prime}$ lying in in the invariable plane pointing along the line of nodes is then

$$
\hat{\mathbf{x}}^{\prime}=(\cos \Omega) \hat{\mathbf{x}}+(\sin \Omega) \hat{\mathbf{y}}
$$

The unit vector from the Sun pointing to the Pluto-Charon system is

$$
\hat{\mathbf{r}}_{\mathbf{s}}=[\cos (\omega+f)] \hat{\mathbf{x}}^{\prime}+[\sin (\omega+f)] \hat{\mathbf{y}}^{\prime}
$$

where $\omega$ is the argument of perihelion and $f$ is the true anomaly.

Inside the Pluto-Charon system let

$$
\hat{\mathbf{b}}=(\sin \theta \cos \phi) \hat{\mathbf{x}}^{\prime}+(\sin \theta \sin \phi) \hat{\mathbf{y}}^{\prime}+(\cos \theta) \hat{\mathbf{n}}
$$

be the unit vector normal to Charon's orbit about Pluto. Here $\theta$ is the obliquity of Charon's orbit and $\phi$ is the east longitude of $\hat{\mathbf{b}}$ on the orbital plane of the Pluto-Charon system about the Sun; see Figure 2. Likewise, the unit spin vector $\hat{\mathbf{s}}$ of Pluto, which points along its axis of rotation, is given by

$$
\hat{\mathbf{s}}=(\sin \Theta \cos \Phi) \hat{\mathbf{x}}^{\prime}+(\sin \Theta \sin \Phi) \hat{\mathbf{y}}^{\prime}+(\cos \Theta) \hat{\mathbf{n}}
$$

where $\Theta$ and $\Phi$ are analogous to $\theta$ and $\phi$, respectively. 
The goal of the following derivation is to find the time derivatives of $d \theta / d t, d \phi / d t$,

$d \Theta / d t$, and $d \Phi / d t$. Clearly

$$
\begin{aligned}
& \cos \theta=\hat{\mathbf{b}} \bullet \hat{\mathbf{n}} \\
& \cos \phi=\hat{\mathbf{x}}^{\prime} \cdot\left[\frac{\hat{\mathbf{b}}-(\hat{\mathbf{b}} \bullet \hat{\mathbf{n}}) \hat{\mathbf{n}}}{\sin \theta}\right] \\
& \cos \Theta=\hat{\mathbf{s}} \bullet \hat{\mathbf{n}} \\
& \cos \Phi=\hat{\mathbf{x}}^{\prime} \bullet\left[\frac{\hat{\mathbf{s}}-(\hat{\mathbf{s}} \bullet \hat{\mathbf{n}}) \hat{\mathbf{n}}}{\sin \Theta}\right]
\end{aligned}
$$

Concentrating on $\theta$ and $\phi$ first, differentiating (20) and (21) yields

$$
\frac{d \theta}{d t}=-\frac{1}{\sin \theta}\left[\hat{\mathbf{b}} \bullet \frac{d \hat{\mathbf{n}}}{d t}+\hat{\mathbf{n}} \bullet \frac{d \hat{\mathbf{b}}}{d t}\right]
$$

and

$$
\frac{d \phi}{d t}=\frac{\cos \theta}{\sin ^{2} \theta \sin \phi}\left(\hat{\mathbf{x}}^{\prime} \bullet \hat{\mathbf{b}}\right)\left(\frac{d \theta}{d t}\right)-\frac{1}{\sin \theta \sin \phi}\left(\hat{\mathbf{b}} \bullet \frac{d \hat{\mathbf{x}}^{\prime}}{d t}+\hat{\mathbf{x}}^{\prime} \bullet \frac{d \hat{\mathbf{b}}}{d t}\right)
$$

where by (16) 
Rubincam $\quad 11$ April 2000

$$
\frac{d \hat{\mathbf{x}}^{\prime}}{\mathrm{dt}}=\frac{d \Omega}{d t}[(-\sin \Omega) \hat{\mathbf{x}}+(\cos \Omega) \hat{\mathbf{y}}]
$$

and by (15)

$$
\begin{aligned}
\frac{d \hat{\mathbf{n}}}{d t}= & \left(\cos I \sin \Omega \frac{d I}{d t}+\sin I \cos \Omega \frac{d \Omega}{d t}\right) \hat{\mathbf{x}} \\
& -\left(\cos I \cos \Omega \frac{d I}{d t}-\sin I \sin \Omega \frac{d \Omega}{d t}\right) \hat{\mathbf{y}} \\
& -\left(\sin I \frac{d I}{d t}\right) \hat{\mathbf{z}}
\end{aligned}
$$

The time rate of change of the orbital angular momentum of Pluto and the rotational angular momentum of Pluto can be written

$$
\begin{aligned}
& \frac{d(h \hat{\mathbf{b}})}{d t}=-L(\hat{\mathbf{s}} \times \hat{\mathbf{b}})(\hat{\mathbf{s}} \bullet \hat{\mathbf{b}})+\frac{3 G M_{S} M_{C} a^{2}}{2 r_{S}^{3}}\left(\hat{\mathbf{r}}_{\mathrm{s}} \bullet \hat{\mathbf{b}}\right)\left(\hat{\mathbf{r}}_{\mathbf{s}} \times \hat{\mathbf{b}}\right)+\mathbf{T}_{\mathbf{c}} \\
& \frac{d(\mathrm{H} \hat{\mathbf{s}})}{d t}=+L(\hat{\mathbf{s}} \times \hat{\mathbf{b}})(\hat{\mathbf{s}} \bullet \hat{\mathbf{b}})+\frac{3 J_{2}^{P} G M_{S} M_{P} R_{P}^{2}}{r_{S}^{3}}\left(\hat{\mathbf{r}}_{s} \bullet \hat{\mathbf{s}}\right)\left(\hat{\mathbf{r}}_{\mathbf{s}} \times \hat{\mathbf{s}}\right)+\mathbf{T}_{\mathbf{P}}
\end{aligned}
$$

where

$$
h=M_{C} \sqrt{G\left(M_{P}+M_{C}\right) a}
$$

and where Charon is assumed to be in a circular orbit about Pluto. Equations (28) and (29) are analogous to Goldreich's [1966] equations (22), where $\hat{\mathbf{s}}$ replaces Goldreich's $\hat{\mathbf{a}}$ and 
$\hat{\mathbf{n}}$ replaces his $\hat{\mathbf{c}}$. The first terms on the right-hand side are the same as Goldreich's: they represent the gravitational attraction between Charon and the equatorial bulge of Pluto, where Charon's motion is averaged over one revolution, so that basically Charon forms a ring. In effect, a ring attracts a ring. The last terms are the tidal torque $T_{C}$ on Charon's orbit and tidal torque $\boldsymbol{T}_{\boldsymbol{P}}$ acting on Pluto. The middle terms give the attraction of the Sun on Charon's ring and the Sun's attraction on Pluto's equatorial bulge when the system is at a distance $r_{s}$ from the Sun. These terms are unlike Goldreich's equations, in that they do not assume that the Pluto-Charon system is in a circular orbit about the Sun. Instead the system is taken to be in an elliptical orbit, which, as has been seen above, is crucial to the eccentricity resonance. These terms are also unlike Ward's [1974], in that they are not averaged over the orbital period.

The derivatives $d \hat{\mathbf{b}} / d t$ and $d \hat{\mathbf{s}} / d t$ can be found from

$$
\frac{d(h \hat{\mathbf{b}})}{d t}=\frac{d h}{d t} \hat{\mathbf{b}}+h \frac{d \hat{\mathbf{b}}}{d t}
$$

so that

$$
\frac{d \hat{\mathbf{b}}}{d t}=-\frac{1}{h} \frac{d(h \hat{\mathbf{b}})}{d t}+\frac{1}{h} \frac{d h}{d t} \hat{\mathbf{b}}
$$

Likewise,

$$
\frac{d \hat{\mathbf{s}}}{d t}=-\frac{1}{H} \frac{d(H \hat{\mathbf{s}})}{d t}+\frac{1}{H} \frac{d H}{d t} \hat{\mathbf{s}}
$$


so that by (28), (29), (32), and (33)

$$
\begin{aligned}
& \frac{d h}{d t}=\mathbf{T}_{\mathrm{c}} \bullet \hat{\mathbf{b}} \\
& \frac{d H}{d t}=\mathbf{T}_{\mathbf{P}} \bullet \hat{\mathbf{s}}
\end{aligned}
$$

It is also helpful to note that

$$
\left(\hat{\mathbf{r}}_{\mathbf{s}} \bullet \hat{\mathbf{b}}\right)\left[\hat{\mathbf{n}} \bullet\left(\hat{\mathbf{r}}_{\mathbf{s}} \times \hat{\mathbf{b}}\right)\right]=\frac{\sin ^{2} \theta}{2} \sin [2 \phi-2(\omega+f)]
$$

and

$$
\begin{gathered}
\frac{1}{r_{s}^{3}}=\frac{1}{a_{s}^{3}} \sum_{q=-\infty}^{+\infty} G_{21 q}(e) \sin (q M) \\
\frac{\sin [2(\omega+f)]}{r_{s}^{3}}=\frac{1}{a_{S}^{3}} \sum_{q=-\infty}^{+\infty} G_{20 q}(e) \sin [2 \omega+(2+q) M] \\
\frac{\cos [2(\omega+f)]}{r_{s}^{3}}=\frac{1}{a_{s}^{3}} \sum_{q=-\infty}^{+\infty} G_{20 q}(e) \cos [2 \omega+(2+q) M]
\end{gathered}
$$

where $a_{s}$ is the semimajor axis, $M$ is the mean anomaly of the Pluto-Charon system's orbit about the Sun, and the $G_{2 p q}(e)$ are the familiar second-degree eccentricity functions from 
geodesy [Kaula, 1966, p. 35; Caputo, 1967, p. 149]. After a great deal of trigonometry (24) and (25) become

$$
\begin{aligned}
\frac{d \theta}{d t}= & -\sin I \cos \phi \frac{d \Omega}{d t}+\sin \phi \frac{d I}{d t} \\
& -\frac{L}{2 h}\left[\sin ^{2} \Theta \sin \theta \sin (2 \Phi-2 \phi)+\sin 2 \Theta \cos \theta \sin (\Phi-\phi)\right] \\
& +\frac{3 G M_{S} M_{C} a^{2}}{4 h} \frac{\sin \theta}{a_{s}^{3}} \sum_{q=-\infty}^{+\infty} G_{20 q}(e) \sin [2 \omega+(2+q) M-2 \Phi] \\
& -\frac{(\hat{\mathbf{n}}-\cos \theta \hat{\mathbf{b}}) \cdot \mathbf{T}_{\mathrm{c}}}{h \sin \theta}
\end{aligned}
$$

$$
\begin{aligned}
\frac{d \phi}{d t}= & (\sin I \cot \theta \sin \phi-\cos I) \frac{d \Omega}{d t}+\cot \theta \cos \phi \frac{d I}{d t} \\
& +\frac{L}{2 h}\left[-2 \cos \theta+3 \sin ^{2} \Theta \cos \theta+\frac{\sin 2 \Theta \cos 2 \theta \cos (\Phi-\phi)}{\sin \theta}+\sin ^{2} \Theta \cos \theta \cos (2 \Phi-2 \phi)\right] \\
& -\frac{3 G M_{S} M_{C} a^{2}}{4 h} \frac{\cos \theta}{a_{S}^{3}} \sum_{q=-\infty}^{+\infty} G_{20 q}(e) \cos [2 \omega+(2+q) M-2 \Phi] \\
& -\frac{3 G M_{S} M_{C} a^{2}}{4 h} \frac{\cos \theta}{a_{S}^{3}} \sum_{q=-\infty}^{+\infty} G_{21 q}(e) \cos (q M) \\
& +\frac{(\hat{\mathbf{n}} \times \hat{\mathbf{b}}) \bullet \mathbf{T}_{\mathrm{c}}}{h \sin ^{2} \theta}
\end{aligned}
$$

From a completely analogous derivation one also gets 


$$
\begin{aligned}
\frac{d \Theta}{d t}= & -\sin I \cos \Phi \frac{d \Omega}{d t}+\sin \Phi \frac{d I}{d t} \\
& +\frac{L}{2 H}\left[\sin ^{2} \theta \sin \Theta \sin (2 \Phi-2 \phi)+\sin 2 \theta \cos \Theta \sin (\Phi-\phi)\right] \\
& +\frac{3 J_{2}^{P} G M_{S}\left(M_{P}+M_{C}\right) R_{P}^{2}}{2 H} \frac{\sin \Theta}{a_{S}^{3}} \sum_{q=-\infty}^{+\infty} G_{20 q}(e) \sin [2 \omega+(2+q) M-2 \Phi] \\
& -\frac{(\hat{\mathbf{n}}-\cos \Theta \hat{\mathbf{s}}) \cdot \mathbf{T}_{\mathbf{P}}}{H \sin \Theta}
\end{aligned}
$$

$$
\begin{aligned}
\frac{d \Phi}{d t}= & (\sin I \cot \Theta \sin \Phi-\cos I) \frac{d \Omega}{d t}+\cot \Theta \cos \Phi \frac{d I}{d t} \\
& -\frac{L}{2 H}\left[2 \cos \Theta-3 \sin ^{2} \theta \cos \Theta-\frac{\sin 2 \theta \cos 2 \Theta \cos (\Phi-\phi)}{\sin \Theta}-\sin ^{2} \theta \cos \Theta \cos (2 \Phi-2 \phi)\right] \\
& -\frac{3 J_{2}^{P} G M_{S}\left(M_{P}+M_{C}\right) R_{P}^{2}}{2 H} \frac{\cos \Theta}{a_{S}^{3}} \sum_{q=-\infty}^{+\infty} G_{20 q}(e) \cos [2 \omega+(2+q) M-2 \Phi] \\
& -\frac{3 J_{2}^{P} G M_{S}\left(M_{P}+M_{C}\right) R_{P}^{2}}{2 H} \frac{\cos \Theta}{a_{S}^{3}} \sum_{q=-\infty}^{+\infty} G_{21 q}(e) \cos (q M) \\
& +\frac{(\hat{\mathbf{n}} \times \hat{\mathbf{s}}) \bullet \mathbf{T}_{\mathrm{P}}}{H \sin \Theta}
\end{aligned}
$$

Equations (40)-(43) are the fundamental equations of the Pluto-Charon system.

Equations (40)-(43) will be simplified in the following ways. The only effect from the tidal torques $T_{C}$ and $T_{P}$ which will be considered here will be the the equilibrium tidal bulges; these will simply be lumped into the $J_{2}^{P}$ coefficient for Pluto. Tidal friction will be dealt with in a future paper. Hence in what follows below 


$$
\frac{d h}{d t}=\frac{d H}{d t}=0
$$

by (28) and (29), so that the magnitudes of Charon's orbit angular momentum $h$ and Pluto's spin angular momentum $H$ remain constant. The $d I / d t$ terms will be ignored, since these are fairly small. Also, only the terms in the $G_{2 p q}(e)$ up to the second power in $e$ will be considered here, so that $q$ takes on only the values $-2,-1,0,+1$, and +2 in the summations.

\section{Volatile migration}

Dobrovolskis et al. [1997], in their own numerical integration, find that Pluto's obliquity and precession angle vary according to the approximate equations

$$
\begin{gathered}
\Theta \approx \theta \approx 115.5^{\circ}+11.8^{\circ} \sin \phi \\
\Phi \approx \phi \approx 19.5^{\circ}+130.2^{\circ} t
\end{gathered}
$$

where $t$ is in millions of years, so that the obliquity varies by about $\pm 12^{\circ}$ with a period of approximately $2.8 \times 10^{6}$ years. This result is confirmed in the integration here. The obliquity oscillation is expected to cause nitrogen and methane to migrate between Pluto's equator and pole as the insolation pattern changes [Spencer et al., 1997]. This transport could cause Pluto's dynamical flattening to change. The volatile loading will also cause viscoelastic relaxation, which in turn will change the flattening. Therefore in (40)-(43) 


$$
J_{2}^{P}=J_{2, h y d r o}^{P}+\Delta J_{2}^{P}
$$

where $J_{2, h y d r o}^{P}$ is given by (6) and $\Delta J_{2}^{P}$ is the change in Pluto's $J_{2}$ coefficient due to the volatiles, elasticity, and viscosity.

Now, the hydrostatic geometric flattening $f_{\text {hydro }}$ is approximately [Kaula, 1968, p. 69]

$$
f_{\text {hydro }} \approx \frac{3}{2} J_{2, h y d r o}^{P}+\frac{\omega_{P}^{2} R_{P}^{3}}{2 G M_{P}}
$$

which gives values on the order of 0.0003 . This gives a $340 \mathrm{~m}$ difference in the polar versus equatorial radius. The amount of volatile transport over an obliquity cycle is unknown, but theoretically could be several kilometers [Spencer et al., 1997]. Thus the theoretical maximum flattening produced by volatiles might be several times greater than the $340 \mathrm{~m}$ produced by hydrostatic flattening. Hence crossing the eccentricity resonance and the half-Plutonian year resonance, which requires doubling $J_{2}^{P}$ from volatile migration, appears to be possible.

The effective viscosity of Pluto's mantle, which depends strongly on temperature, is unknown [McKinnon et al., 1997]. Marcialis [1985] has argued that the relaxation time could be as short as 30,000 years, producing a topographically bland planet. It will be assumed here that Pluto can support nonhydrostatic loads for long periods of time.

\section{Numerical integration}

The values used in the numerical integrations are listed in Table 1 . The inclination $I$ of orbit about the Sun with respect to the invariable plane, its argument of perihelion $\omega$, and 
nodal rate $d \Omega / d t$ were all assumed to be constant. The starting values for $\theta, \phi, \Theta$, and $\Phi$ (denoted by the subscript zero in Table 1) were taken from (45) and (46) for $t=0$, so that initially Charon orbits in Pluto's equatorial plane. The other values for the Pluto-Charon system shown in Table 1 were somewhat arbitrary, but chosen to insure that Pluto crossed the eccentricity resonance as its flattening changed.

The change in flattening due to the movement of volatiles was assumed to have one of the two forms

$$
\begin{aligned}
& \frac{\Delta J_{2}^{P}}{J_{2, \text { hydro }}^{P}}=-F \sin \phi \\
& \frac{\Delta J_{2}^{P}}{J_{2, \text { hydro }}^{P}}=+F \cos \phi
\end{aligned}
$$

so that by (45) the change was proportional to the change in obliquity of the Pluto-Charon system. In the case of (49) there is no phase lag, and in the case of (50) the phase lag is $90^{\circ}$. The negative sign in (49) comes from Pluto having a mean obliquity wherein the average annual insolation at the poles is greater than at the equator [Ward, 1974, Figure 9]; as the obliquity oscillates to a higher value, the poles see less sunlight, so that volatiles would be expected to accumulate there, leading to a smaller $J_{2}^{P}$. The rationale for the $90^{\circ}$ phase lag in (50) is that this is what would be expected on a volatile-rich planet [Rubincam, 1995, p. 371]. $F$ is simply the maximum fractional change in $J_{2}^{P}$ compared to hydrostatic equilibrium. $F$ arbitrarily took on the values $0.016,0.167$, and 1.67 in the integrations. The change in Pluto's $J_{2}^{P}$ in geared through $\phi$ to the obliquity $\theta$ of Charon's tilt, rather than though Pluto's obliquity $\Theta$. The reason is that $\phi$ provides a smoother changing flattening, 
since the smaller angular momentum of Pluto compared to Charon's orbit can cause $\Theta$ can jitter on a rapid timescale. For runs where $F=1.67, J_{2}{ }^{P}$ was not allowed to drop below $15 \%$ of its hydrostatic value, the reason being that a larger accumulation of volatiles at the poles would cause polar wander, limiting the size of $J_{2}^{P}$. Polar wander on Pluto will be treated in a future paper.

David D. Rowlands wrote the program which integrated (40)-(43) according to an eleventh order Cowell predictor-corrector scheme. Runs were made on a Cray YMP supercomputer. The step size was $9.467 \times 10^{6}$ seconds for $F=0.0167$ and 0.167 . To integrate through one complete obliquity oscillation required about $9,400,000$ steps. The same step size was used for $F=1.67$, except in the neighborhood of the resonances, where the integrations with this step size were unstable. In these regions the step size was decreased by a factor of 50 .

The results are shown in Figures 3-4. Both figures show the angle between Pluto's equator and Charon's orbital plane $I_{P C}$ as a function of time with high-frequency oscillations smoothed out, where

$$
\cos I_{P C}=\hat{b} \bullet \hat{s}
$$

In other words, $I_{P C}$ is the mutual inclination. Figure 3 (solid line) uses (49) with $F=$ 0.0167 only, while Figure 4 uses (50) with $F=0.0167$ (solid line), 0.167 (dashed line), and 1.67 (dotted line).

In the case of $F=0.0167$ (solid lines in Figures 3 and 4) Pluto's geometric flattening changes by about $10 \mathrm{~m}$. Each graph shows that Charon's orbital plane can tilt out of Pluto's equatorial plane by as much as $2^{\circ}$ by crossing the eccentricity resonance for this value of $F$, assuming there is no tidal friction. The change in flattening for this value 
of $F$ is not large enough for Pluto to cross the half-Plutonian year resonance. In Figure 3 $I_{P C}$ immediately jumps ups to a nonzero value.

Using (50) with $F=0.167$ (dashed line in Figure 4) is also not large enough to cross the half-Plutonian year resonance. In this case $I_{P C}$ is only about $0.3^{\circ}$ after $5.6 \times 10^{6}$ years. This is smaller than for $F=0.0167$, so it must be concluded that the size of the jumps depends on the speed at which Pluto crosses the resonance. A large change in flattening over the obliquity cycle means that Pluto spends little time near the eccentricity resonance, producing smaller jumps.

Using (50) with $F=1.67$ is shown by the dotted line in Figure 4. Here the change in Pluto's flattening is now large enough to cross the half-Plutonian year resonance, which it does near $0.25 \times 10^{6}, 2 \times 10^{6}, 2.8 \times 10^{6}$, and $4.8 \times 10^{6}$ years. The increase in $I_{P C}$ after $5.6 \times 10^{6}$ years is larger than for the case where $F=0.167$, thanks to crossing the strong half-Plutonian year resonance. Of course Pluto still crosses the eccentricity resonance, but these crossings produce negligible changes in mutual inclination.

Other computer runs were made with no volatile migration at all $\left(\Delta J_{2}^{P}=0\right)$, so that $J_{2}^{P}=$ constant. $J_{2}^{P}$ was then adjusted to make the system as resonant as possible. This lead to $I_{P C}$ oscillating to values as large as $4^{\circ}$. From this result it is not surprising that the smallest amount of volatile movement ( $F=0.0167$ in Figures 3 and 4$)$ produces the largest jumps: the parameters have been chosen to insure that the system crosses the resonance; a small amount of volatiles means the system is already very close to being resonant, producing large changes in $I_{P C}$.

\section{Tidal friction and libration}


While foregoing computations did not include tidal friction, its effect on the mutual inclination $I_{P C}$ can be estimated from the tidal friction equation of Kaula [1964, p. 676]:

$$
\begin{aligned}
\frac{d I_{P C}}{d t} & =-k_{2}\left|\sin \varepsilon_{2100}\right|\left(\frac{R_{P}}{a}\right)^{5} \frac{G M_{C}}{3 n a^{3} \sin I_{P C}}\left[F_{211}\left(I_{P C}\right)\right]^{2} \\
& \approx-\frac{3}{4} n\left(\frac{M_{C}}{M_{P}+M_{C}}\right)\left(\frac{R_{P}}{a}\right)^{5}\left(\frac{k_{2}}{Q}\right) I_{P C}
\end{aligned}
$$

where only the $\ell=2, m=1, p=1, q=0$ term gives a significant contribution, with the $F_{\text {emp }}\left(I_{P C}\right)$ being the inclination functions, $k_{2}$ the Love number, $\sin \varepsilon_{2110} \approx 1 / Q(Q$ being the quality factor), and $\sin I_{P C} \approx I_{P C}$. For the parameters of the system given in Table 1 (52) can be easily integrated to give

$$
I_{P C}=I_{P C}^{0} e^{-23.6\left(k_{2} / Q\right) t}
$$

where $t$ is in millions of years, so that tidal friction operating alone makes the mutual inclination decay towards zero. The ratio $k_{2} / Q$ is unknown for Pluto; [Dobrovolskis et al., 1997 , p. 179] estimate may $k_{2}$ may be as high as 0.053 , while $Q$ is usually assumed to be about 100, so that $k_{z} / Q<0.00053$. Goldreich et al. [1989] assume $k_{z} / Q=10^{-3}$ for Triton, which is similar in size and composition to Pluto. Using Goldreich et al.'s higher value as the worst case estimate gives a decay in $I_{P C}$ of only $7 \%$ over the $3 \times 10^{6}$ year precession cycle, so that it does not appear that tidal friction would damp out the resonance jumps very much for the times considered above, justifying its neglect in the integrations here. It could become a major factor on this short timescale if $k_{z} / Q$ is a not-impossible 10 times larger 
than estimated. Tidal friction will definitely have to be taken into account for longer periods of time (see the Discussion) than just the two obliquity cycles examined above.

Libration has also been ignored here. In addition to rigid body libration, there will be libration due to volatile migration as well. The movement of volatiles will change Pluto's moment of inertia, so that its spin rate will change due to conservation of angular momentum. Pluto's rotation will no longer be synchronous, and the tidal bulge raised by Charon on Pluto will swing out of the line joining their centers until checked by Charon's torque and synchronism is reestablished. The torque will alter Pluto's rotation rate and its distance from Charon. These phenomena are not included in the above calculations and represent future avenues for research.

\section{Discussion}

The foregoing analysis indicates that Pluto may cross the eccentricity resonance and possibly the half-Plutonian year resonance through the movement of volatiles during the 3 $\times 10^{6}$ obliquity oscillation. The integrations over two precession cycles indicate that the Charon's orbital plane may depart from Pluto's equatorial plane by as much as $2^{\circ}$, depending on the supply of volatiles and how close Pluto is to the resonance. Because the integrations were not carried past $5.6 \times 10^{6}$ years, it is not presently known how large the mutual inclination $I_{P C}$ might grow with time. Given that making the system as resonant as possible gives at most $4^{\circ}$, the maximum tilt may be $4^{\circ}$, but longer integrations are necessary to settle the question. Though tidal friction was ignored here, it will have to be taken into account for longer periods of time, so that the mutual inclination over the age of the solar system will be controlled by the interplay of tidal friction and resonance crossing. Observing that Charon orbits out of Pluto's equatorial plane would be evidence for 
resonance crossing and the movement of volatiles, but if the mutual tilt is only a few degrees, its detection would be difficult from just a flyby mission.

A Pluto flyby mission might be expected to measure $M_{P}, M_{C}, R_{P}, J_{2}^{P}, a, n$, and $\omega_{\mathrm{p}}$. If Pluto is in hydrostatic equilibrium, then $J_{2}^{p}$ provides the condensation factor $\lambda_{\mathrm{p}}$ via (6), (8), and (10). However, the migration of volatiles may cause Pluto's flattening to be nonhydrostatic, spoiling the determination of the moment of inertia. A measurement of the mutual tilt might at least provide a weak constraint between $\lambda_{p}$, the movement of volatiles, and tidal friction.

The migration of volatiles and the subsequent resonance jumps may be a way of secular changing Pluto's obliquity through the phenomenon of climate friction [e.g., Rubincam, 1990, 1993, 1995, 1999], also known as obliquity-oblateness feedback [Bills, 1999]. This will be investigated in a future paper.

The question arises as to whether Pluto is not just crosses, but actually formed in the eccentricity resonance. There are strong arguments against it. There is no obvious reason why Pluto should be in the resonance. Pluto and Charon reach their final Cassini states within a few million years of formation [Farinella et al., 1979]; the states are determined by the total angular momentum of the system. If the resonance primordial, by what process did the masses apportion themselves in the correct ratio during accretion so as to fall in the resonance? And why should it fall into it? With Charon out of the equatorial plane, tidal friction would dissipate energy. One would expect a final state in which energy dissipation is minimized, not maximized. Even if the system did form in the resonance, impacts might be expected to disturb it away from the resonant state.

Another argument, due to Bruce G. Bills (private communication, 1999), is the narrowness of the resonance: a deviation of Pluto's flattening of only a meter or two away from the resonant value would cut the tilt from $4^{\circ}$ to a fraction of a degree. That Pluto's 
shape would conform to such a narrow tolerance seems unlikely. Still, it is a peculiar coincidence that the Pluto-Charon system is so close to the eccentricity resonance. There may be some unthought-of mechanism that forces the system into it.

Acknowledgments. I thank David D. Rowlands, Sue Blackwell, and Susan Poulose for excellent programming support. I thank Bruce G. Bills for discussions, particularly for pointing out that the resonances might be narrow. I thank Katy Gammage, Jay O'Leary, and Brad Lee for producing the figures. 


\section{References}

Bills, B. G., Obliquity-oblateness feedback on Mars, J. Geophys. Res., 104, 30,773$30,797,1999$.

Brouwer, D. and G. M. Clemence, Methods of Celestial Mechanics, Academic Press, New York, 1961.

Bursa, M., Tidal and rotational distortions in figures of Pluto and Charon, Earth Moon Planet., 65, 291-294, 1994.

Caputo, The Gravity Field of the Earth, Academic Press, New York, 1967.

Dobrovolskis, A. R., S. J. Peale, and A. W. Harris, Dynamics of the Pluto-Charon binary, in Pluto and Charon, edited by S. A. Stern and D. J. Tholen, pp. 159-190, University of Arizona Press, Tucson, 1997.

Farinella, P., A. Milani, A. M. Nobili, and G. B. Valsecci, Tidal evolution and the PlutoCharon system, Moon Planet., 20, 415-421, 1979.

Goldreich, P., History of the lunar orbit, Rev. Geophys., 4, 411-439, 1966.

Goldreich, P., N. Murray, P. Y. Longaretti, and D. Banfield, Neptune's story, Science, 246, 500-504, 1989.

Kaula, W. M., Tidal dissipation by solid friction and the resulting orbital evolution, Rev. Geophys., 2, 661-685, 1964. 
Kaula, W. M., Theory of Satellite Geodesy, Blaisdell, Waltham, Mass., 1966.

Kaula, W. M., An Introduction to Planetary Physics, Wiley, New York, 1968.

Malhotra, R., and J. G. Williams, Pluto's heliocentric orbit, in Pluto and Charon, edited by S. A. Stern and D. J. Tholen, pp. 127-157, University of Arizona Press, Tucson, 1997.

Marcialis, R. L., Topographic relaxation on ice-covered worlds: Application to Pluto (abstract), Bull. Am. Astron. Soc., 17, 715, 1985.

McKinnon, W. B., D. P. Simonelli, and G. Schubert, Composition, internal structure, and thermal evolution of Pluto and Charon, in Pluto and Charon, edited by S. A. Stern and D. J. Tholen, pp. 295-343, University of Arizona Press, Tucson, 1997.

Null, G. W., and W. M. Owen, Charon/Pluto mass ratio obtained with HST CCD observations in 1991 and 1993, Astron. J., 111, 1368-1381, 1996.

Null, G. W., W. M. Owen, and S. P. Synnott, Masses and densities of Pluto and Charon, Astron. J., 105, 2319-2335, 1993.

Rubincam, D. P., Mars: change in axial tilt due to climate?, Science, 248, 1990.

Rubincam, D. P., The obliquity of Mars and "climate friction," J. Geophys. Res., 98, 10,827-10,832, 1993. 
Rubincam, D. P., Has climate changes the Earth's tilt?, Paleoceanography, 10, 365-372, 1995.

Rubincam, D. P., Mars secular obliquity change due to water ice caps, J. Geophys. Res., 104, 30,765-30,771, 1999.

Spencer, J. R., J. A. Stansberry, L. M. Trafton, E. F. Young, R. P. Binzel, and S. K. Croft, Volatile transport, seasonal cycles, and atmospheric dynamics on Pluto, in Pluto and Charon, edited by S. A. Stern and D. J. Tholen, pp. 435-473, University of Arizona Press, Tucson, 1997.

Tholen, D. J., and M. W. Buie, Bulk properties of Pluto and Charon, in Pluto and Charon, edited by S. A. Stern and D. J. Tholen, pp. 193-219, University of Arizona Press, Tucson, 1997.

Tholen, D. J., and E. F. Tedesco, Pluto's lightcurve: results from four oppositions, Icarus, 108, 200-208, 1994.

Ward, W. R., Climatic variations on Mars, 1, Astronomical theory of insolation, $J$. Geophys. Res., 79, 3375-3386, 1974.

Young, L. A., C. B. Olkin, J. L. Elliot, D. J. Tholen, and M. W. Buie, The Charon-Pluto mass ratio from MKO astrometry, Icarus, 108, 219-224, 1994. 


\section{FIGURE CAPTIONS}

\section{Figure 1}

The precession-orbit resonances, assuming hydrostatic flattening for Pluto. The lower shaded area shows the resonance with the orbital period of Pluto about the Sun (250.8 Earth years), while the upper shaded area shows the other resonance with half the orbital period (125.4 Earth years). For each resonance the lower boundary is at $\left(a / R_{P}\right)=$ 16.2, while the upper boundary is at 17.1. The resonances fall somewhere in the shaded areas. The region between the dashed lines gives the observed range of $M_{C} M_{p}$. Pluto may in the eccentricity resonance, while the half-Plutonian year resonance appears to be ruled out, unless Pluto has a large nonhydrostatic flattening or the mass ratio is seriously in error.

\section{Figure 2}

Diagram of the geometry of the Pluto-Charon-Sun system. The unit vectors $\hat{\mathbf{x}} \hat{\mathbf{y}} \hat{\mathbf{z}}$ forms a right-handed inertial coordinate system, with $\hat{\mathbf{z}}$ being normal to the invariable plane. The unit vector $\hat{\mathbf{n}}$ is normal to the orbital plane of Pluto about the Sun and makes an angle $I$ with $\hat{\mathbf{z}}$. The ascending nodal line AB of Pluto's orbital plane makes an angle $\Omega$ in the invariable plane. Perihelion position is denoted by $\omega$ and the true anomaly by $f$.

Pluto's distance from the Sun is $r_{s}$. The unit vector $\hat{\mathbf{b}}$ is normal to Charon's orbital plane. Charon's orbital plane is tipped by an angle $\theta$ to Pluto's orbital plane about the Sun. The east longitude $\phi$ of $\hat{\mathbf{b}}$ in Pluto's orbital plane is measured from $\mathrm{CD}$, which is parallel to $\mathrm{AB}$. For Pluto's orientation, $\hat{\mathbf{s}}, \Theta$, and $\Phi$ are completely analogous to $\hat{\mathbf{b}}, \theta$, and $\phi$, respectively. The diagram is not to scale. 


\section{Figure 3}

The angle $I_{P C}$ between Pluto's equatorial plane and Charon's orbital plane as a function of time for $\left(\Delta J_{2} / J_{2}^{P}\right)=-F \sin \phi$, where $F=0.0167$. The change in the flattening is in phase with the obliquity oscillations. The initial conditions are $\phi, \Phi=19.5^{\circ}$ and $\theta, \Theta$ $=119.439^{\circ}$, so that Charon starts out orbiting in Pluto's equatorial plane. The jumps occur at the eccentricity resonance, except for the one near $t=0$. The half-Plutonian year resonance is not reached. The $5.6 \times 10^{6}$ year integration gives two complete precession periods of Charon's orbit. The curve is highly smoothed. No tidal friction is assumed.

\section{Figure 4}

The angle $I_{P C}$ between Pluto's equatorial plane and Charon's orbital plane as a function of time for $\left(\Delta J_{2} / J_{2}^{P}\right)=F \cos \phi$, where $F=0.0167$ (solid line), $F=0.167$ (dashed line), and $F=1.67$ (dotted line). The change in flattening is $90^{\circ}$ out of phase with the obliquity oscillations. The initial conditions are $\phi, \Phi=19.5^{\circ}$ and $\theta, \Theta=119.439^{\circ}$, so that Charon starts out orbiting in Pluto's equatorial plane. Only the curve for $F=1.67$ reaches the half-Plutonian year resonance. The $5.6 \times 10^{6}$ year integration gives two complete precession periods of Charon's orbit. These curves are highly smoothed. No tidal friction is assumed. 


\section{TABLE}

\section{Table 1}

Assumed parameters for the Pluto-Charon system. The last four values are the starting values used in the integrations.

$\begin{array}{lll}\mathrm{M}_{\mathrm{p}} & \text { Pluto mass } & 1.288 \times 10^{22} \mathrm{~kg} \\ \mathrm{M}_{\mathrm{C}} & \text { Charon mass } & 1.891 \times 10^{21} \mathrm{~kg} \\ \mathrm{R}_{\mathrm{p}} & \text { Pluto radius } & 1149.825 \mathrm{~km} \\ \lambda_{\mathrm{p}} & \text { condensation factor } & 0.38 \\ \mathrm{a} & \text { Pluto-Charon distance } & 19,662 \mathrm{~km} \\ \mathrm{~d} \Omega / \mathrm{dt} & \text { nodal rate } & -5.37 \times 10^{-14} \mathrm{~s}^{-1} \\ \mathrm{I} & \text { orbital inclination } & 15.91^{\circ} \\ \omega & \text { argument of perigee } & 90^{\circ} \\ \theta_{0} & \text { Charon obliquity } & 119.439^{\circ} \\ \phi_{0} & \text { Charon precession angle } & 19.5^{\circ} \\ \Theta_{0} & \text { Pluto obliquity } & 119.439^{\circ} \\ \Phi_{0} & \text { Pluto precession angle } & 19.5^{\circ}\end{array}$




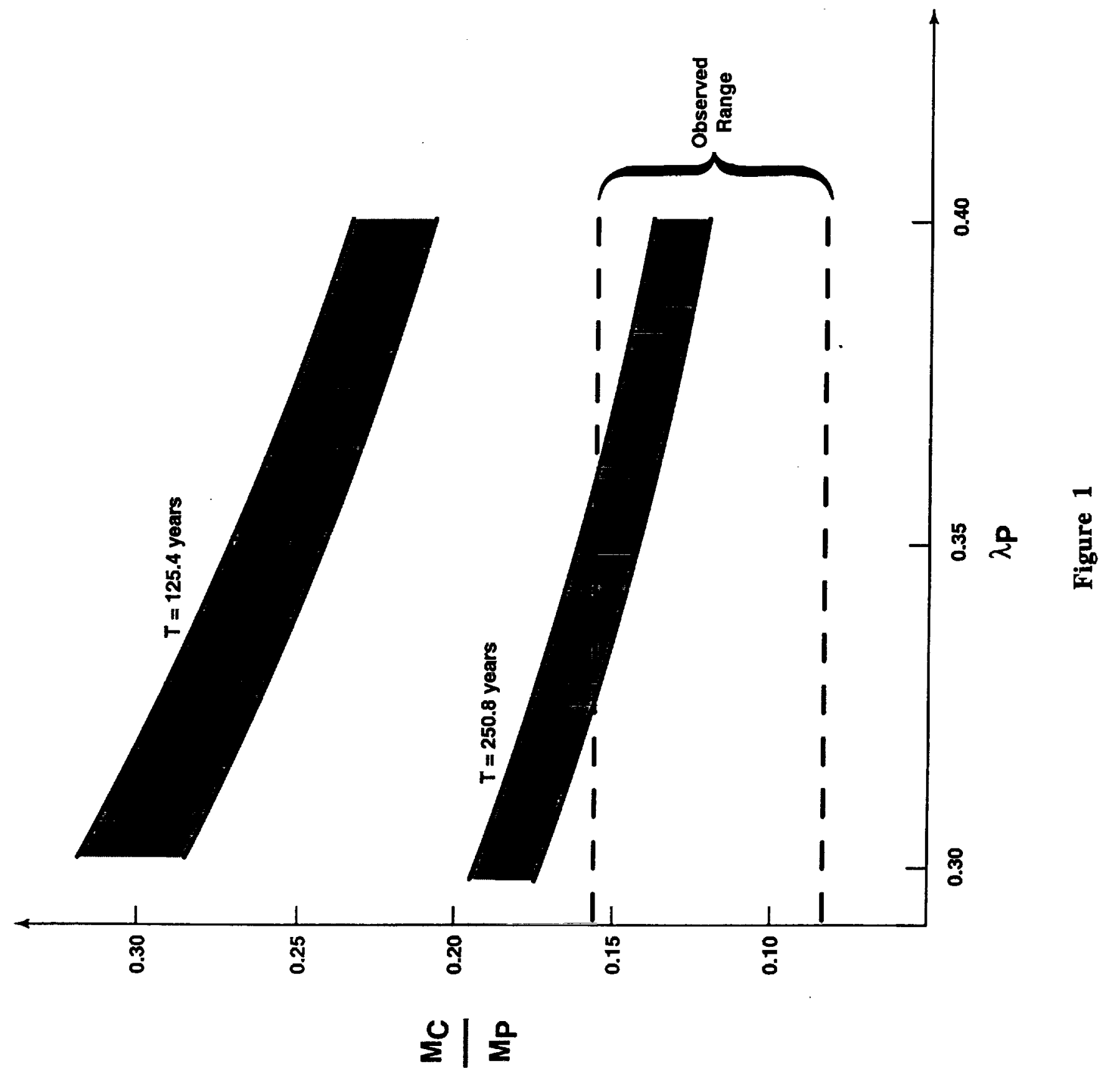




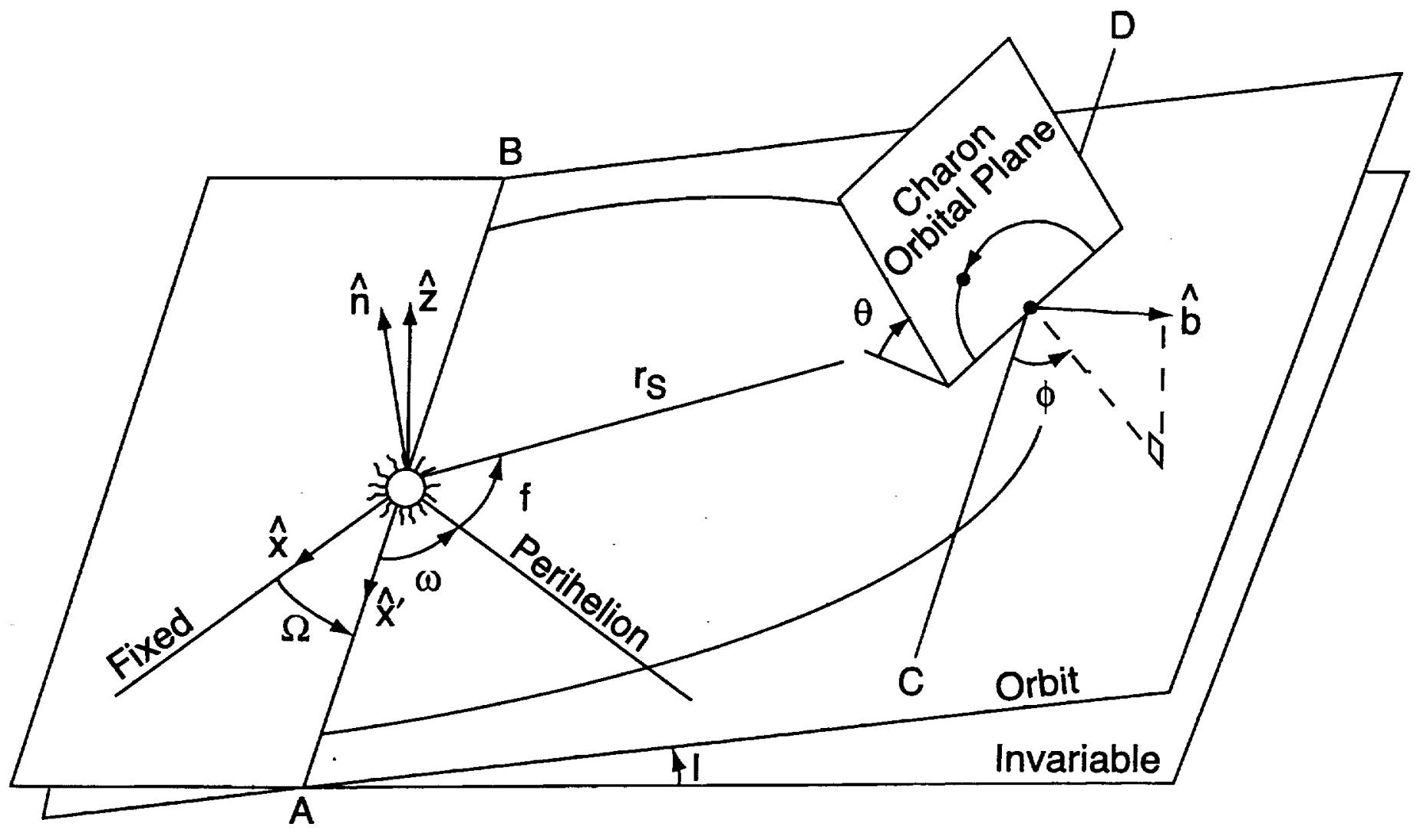

Figure 2 


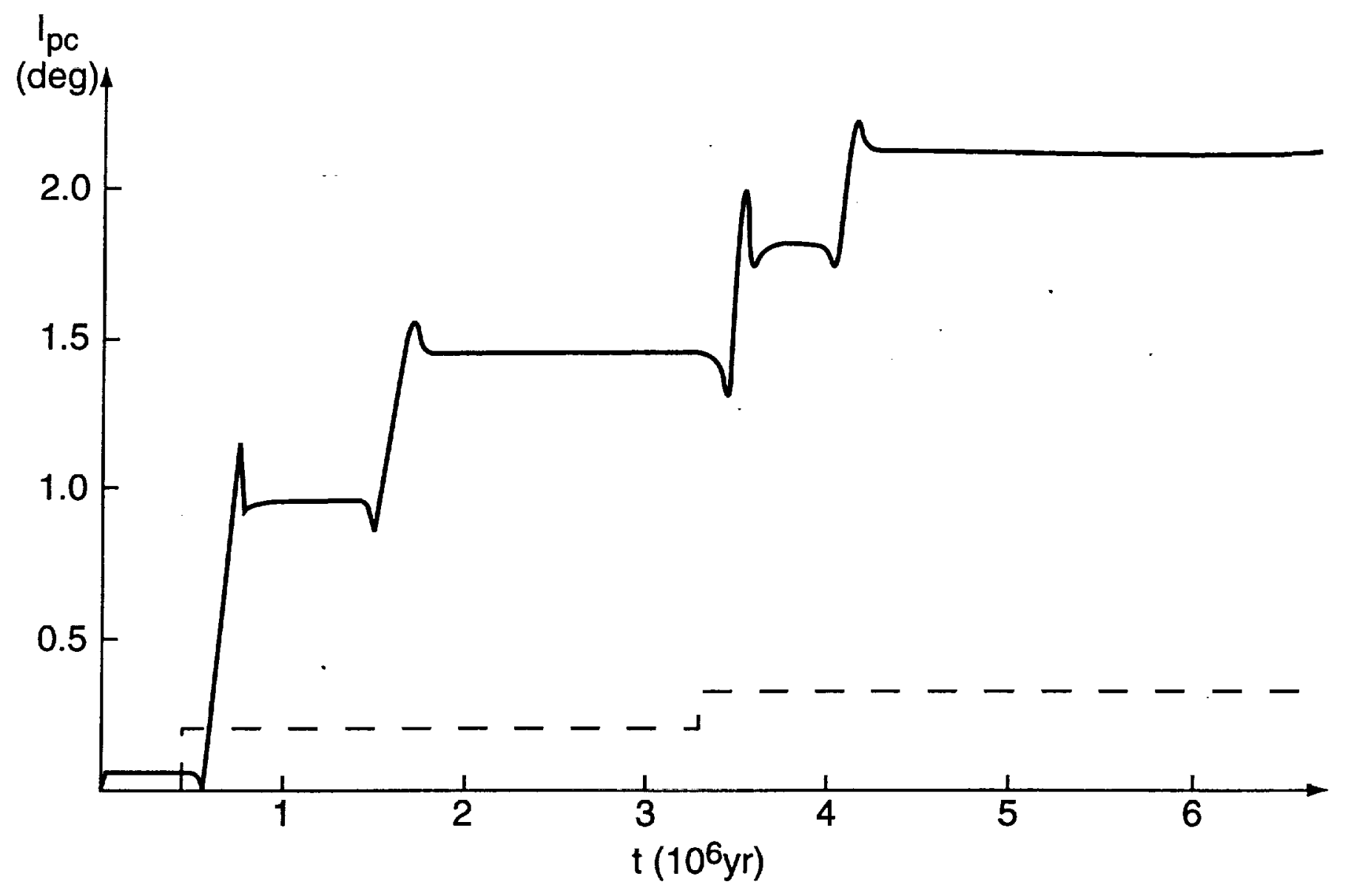

Figure 3 


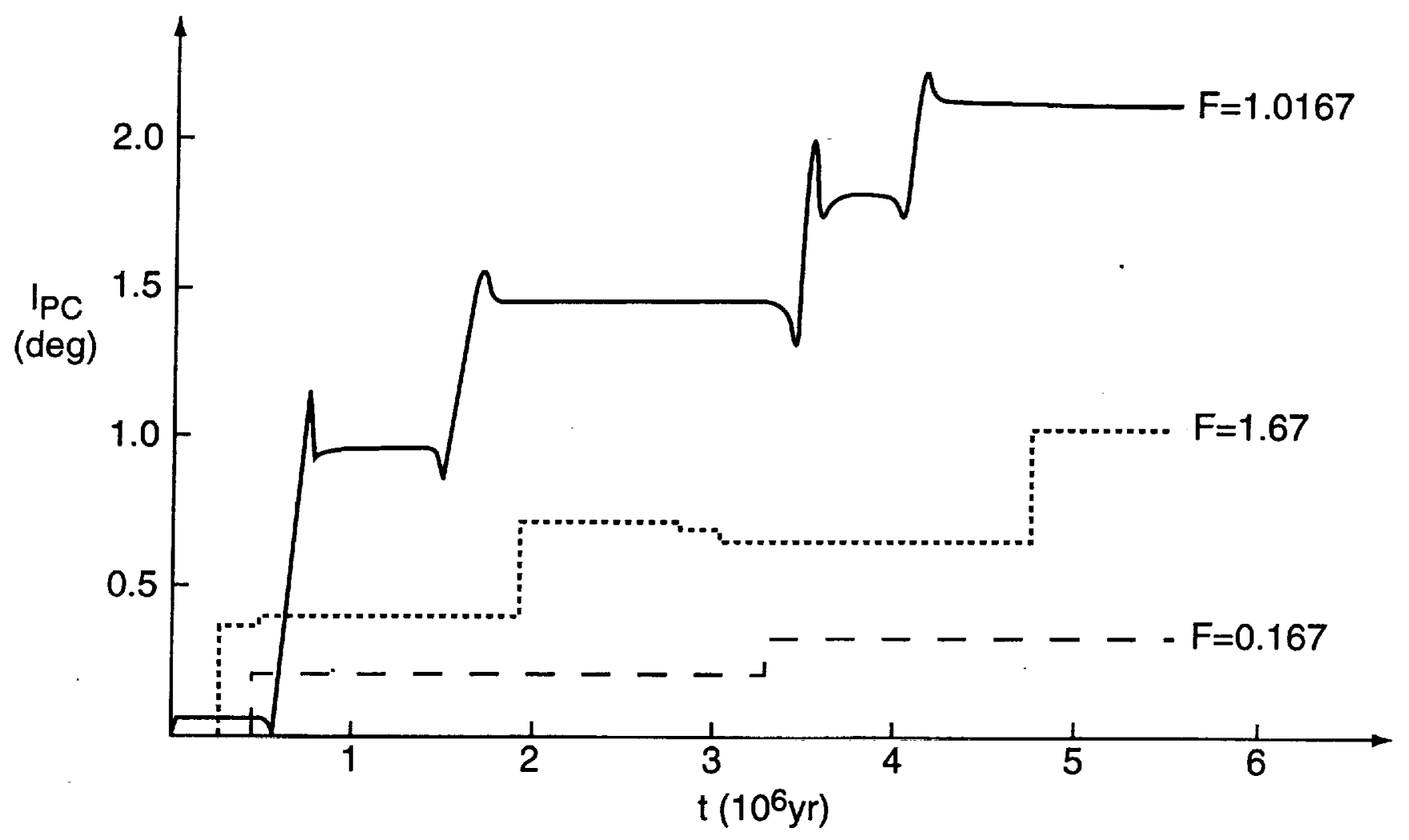

Figure 4 